\title{
ON THE VIBRATIONS OF A HETEROGENEOUS STRING*
}

\section{BY J. L. SYNGE (Dublin Institute for Advanced Studies)}

\begin{abstract}
It is shown that the vibrations of a heterogeneous string can be represented by an infinite series, each term of which is the result of applying a linear integral operator to a function of position and time furnished by the initial data. The method applies also to plane waves of compression or shear in a heterogeneous elastic solid for which the elastic constants and density are functions of only one coordinate and the waves move in the direction of that coordinate.
\end{abstract}

1. The partial differential equation. Consider the partial differential equation

$$
u_{t t}-s^{2} u_{x x}=0
$$

here $s$ is a positive function of $x$ only, $s(x)$. Let $x$ range from $-\infty$ to $+\infty$. The characteristics are given by $d x / d t= \pm s$, which equations define two congruences of curves in the $(x, t)$-plane. The function $s(x)$ represents the local speed of propagation, and the theory applies to all physical systems essentially involving only one space-dimension for which this speed is known. Thus

(1) For the transverse vibrations of a string, $s^{2}=T / \lambda$ where $T$ is the (constant) tension and $\lambda$ the mass per unit length at the position $x$.

(2) For the compressional vibrations of an isotropic elastic solid in which the density and elastic constants are functions of $x$ only (we might call it laminated), $s^{2}=(\lambda+2 \mu) / \rho$.

(3) For the transverse vibrations of such a laminated solid, $s^{2}=\mu / \rho$.

Other applications may occur to the reader; the essential condition is that (1.1) is applicable, with $s(x)$ a given positive function.

2. Straightening the characteristics. The first step is to straighten the characteristics of (1.1) for geometrical representation in a space-time plane. To do this we define

$$
y=\int_{0}^{x} d z / s(z)
$$

giving $y$ as a function of $x$. Inverting, we get $x$ as a single-valued function of $y$, and this gives

$$
s(x)=c(y)
$$

we may call $c(y)$ the transformed local speed. Changing the independent variable from $x$ to $y$, we transform (1.1) into

$$
u_{t t}-u_{y y}+\left(c^{\prime} / c\right) u_{y}=0
$$

* Received February 22, 1980. 
where the prime means $\partial / \partial y$. In a space-time plane in which $y$ and $t$ are Cartesian coordinates, the characteristics are now straight lines inclined to the axes at $45^{\circ}$.

Strictly speaking, we should not use the same symbol $u$ in (1.1) and (2.3), because the function $u(x, t)$ of $(1.1)$ is not the same function as the $u(y, t)$ of $(2.3)$; but this is not likely to cause confusion if we remember that $u$ may be regarded as a physical quantity expressible in terms of $(x, t)$ or $(y, t)$.

3. Changing the dependent variable. Let us see what happens to Eq. (2.3) if we change the dependent variable $u$ to $v$ by the transformation

$$
u(y, t)=v(y, t) \phi(y),
$$

where the function $\phi$ is at present unspecified. Then

$$
u_{t t}=v_{t t} \phi, \quad u_{y}=v_{y} \phi+v \phi^{\prime}, \quad u_{y y}=v_{y y} \phi+2 v_{y} \phi^{\prime}+v \phi^{\prime \prime},
$$

and when these are substituted in (2.3) we get, on division by $\phi$,

$$
\begin{gathered}
v_{t t}-v_{y y}=2 k v_{y}+2 h v, \\
2 k=2 \phi^{\prime} / \phi-c^{\prime} / c, \quad 2 h=\phi^{\prime \prime} / \phi-\left(\phi^{\prime} / \phi\right)\left(c^{\prime} / c\right) .
\end{gathered}
$$

Choose $\phi$ to make $k=0$ :

$$
\phi=c^{1 / 2}, \quad u=v c^{1 / 2}
$$

Then

where

$$
2 h=-\frac{1}{2} c^{\prime \prime} / c+\frac{3}{4}\left(c^{\prime} / c\right)^{2}=-\gamma^{\prime}+\gamma^{2}
$$

$$
\gamma=\frac{1}{2} c^{\prime} / c
$$

Our partial differential equation now reads

$$
v_{t t}-v_{y y}=2 h v
$$

the characteristics are straight and, instead of the partial derivative $u_{y}$ as in (2.3), we now have the function $v$ itself.

4. The integral equation and its solution. If the right-hand side of (3.6) were a given function of $(y, t)$, we would have the equation for a uniform string acted on by external force and we would know how to proceed. This suggests a similar procedure, leading to an integral equation for $v$.

In Fig. $1, \mathbf{P}$ is a general point $(y, t)$, chosen with $t$ positive for convenience, and $\mathrm{A}, \mathrm{B}$ are the points where the characteristics through $\mathrm{P}$ meet the $y$-axis. Integrating (3.6) over the triangle $\mathrm{PAB}$, we get

$$
\begin{aligned}
0 & =\iint\left(v_{y y}-v_{t t}+2 h v\right) d y d t \\
& =\dot{\oint}\left(v_{y} d t+v_{t} d y\right)+2 \iint h v d y d t,
\end{aligned}
$$




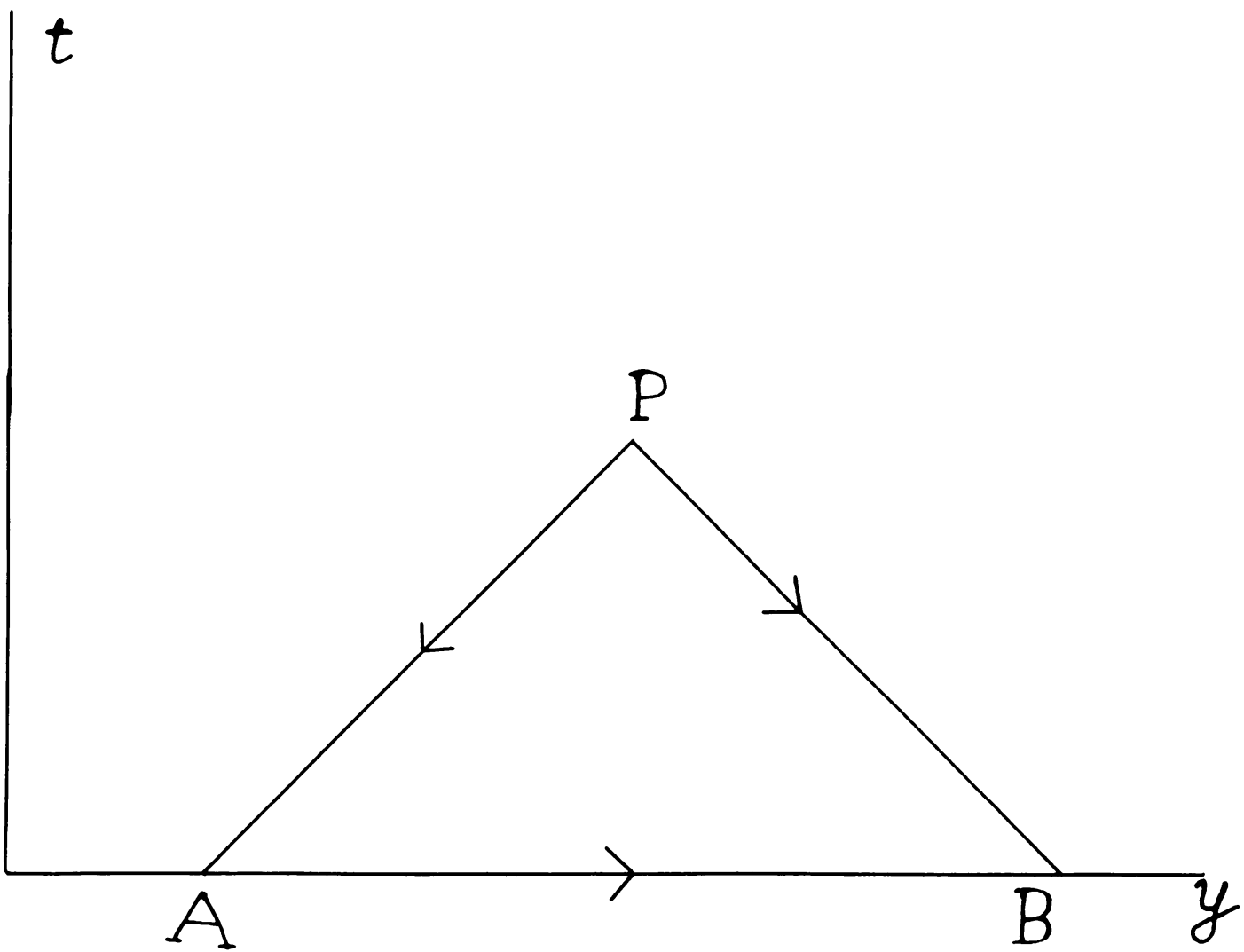

Fig. 1.

the first of these going counterclockwise round $\mathrm{PAB}$. But $d t=d y$ on $\mathrm{PA}$ and $d t=-d y$ on BP. Thus (4.1) gives, in an obvious notation,

$$
v_{\mathrm{A}}-v_{\mathrm{P}}+\int_{\mathrm{AB}} v_{t} d y-v_{\mathrm{P}}+v_{\mathrm{B}}+2 \iint h v d y d t=0,
$$

or

$$
v_{\mathbf{P}}=\frac{1}{2}\left(v_{\mathbf{A}}+v_{\mathbf{B}}+\int_{\mathbf{A B}} v_{t} d y\right)+\iint h v d y d t .
$$

Notationally, the above argument is defective in that, although $I$ said that $\mathbf{P}$ was a given point with coordinates $(y, t)$, I have used $(y, t)$ as current coordinates. To clarify this, let me change the notation for current coordinates, although I expect that the reader might be satisfied with the derivation of (4.2).

Let $(y, t)$ be the coordinates of any point in the $(y, t)$-plane, and $v(y, t)$ any function which satisfies the p.d.e. (3.6). Define

$$
C(y, t)=\frac{1}{2}\left\{v(y-t, 0)+v(y+t, 0)+\int_{y-t}^{y+t} v_{t}(z) d z\right\} ;
$$


this may be called the Cauchy function, since it involves only the usual Cauchy data, viz. the values of $v$ and $v_{t}$ for $t=0$; it satisfies

$$
C_{t t}-C_{y y}=0 \text {. }
$$

Now define the linear integral operator $H$ by the statement that, when applied to an arbitrary function $w(y, t)$, we have

$$
H w(y, t)=\iint h\left(y_{1}\right) w\left(y_{1}, t_{1}\right) d y_{1} d t_{1},
$$

with integration over the characteristic triangle with vertex at $(y, t)$. It must be understood that the combination $H w$ is itself a functional symbol, and $(y, t)$ indicates the point at which that function $H w$ is to be evaluated.

With these definitions, it should be clear that (4.2) may be written

$$
v(y, t)=C(y, t)+H v(y, t)
$$

the function $C$ being determined by the Cauchy data, this is an integral equation for the solution $v(y, t)$.

To solve this integral equation, we write it

$$
(I-H) v=C
$$

where $I$ is the identity operator, multiply by $(I-H)^{-1}$, and expand this binomially. Thus

$$
v=(I-H)^{-1} C=\left(I+H+H^{2}+H^{3}+\cdots\right) C .
$$

This calls for an infinite sequence of operations on the (given) Cauchy function $C(y, t)$. The series converges if $C$ and $h$ are bounded in absolute value, being, as is easily seen, dominated termwise by the exponential series with $n$th term

$$
\bar{C} \hbar^{n} t^{2 n} /(2 n !)
$$

where the bars indicate the upper bounds.

The problem of the heterogeneous string is thus solved, and with it those other problems to which the p.d.e. (1.1) applied. We must however remember that $v$ as in (4.8) is not the physical displacement $u$, but is related to it by $u=v c^{1 / 2}$.

5. An interesting special case. So far we have regarded the function $s(x)$, or equivalently $c(y)$, as arbitrarily given. If we take $s=$ const., we have the d'Alembert solution

$$
u=f(t-x / s)+g(t+x / s)
$$

the two functions being arbitrary until determined by Cauchy data. To get $v$, we simply divide by the constant $c^{1 / 2}$, which equals $s^{1 / 2}$. Can we design a heterogeneous string [i.e. assign $s(x)$ ] so that the general solution of the p.d.e. (1.1) can be expressed in terms of arbitrary functions?

Returning to (3.6), which is nothing but a transform of (1.1) without involving the initial data, we note that if $h=0$ our equation is

$$
v_{t t}-v_{y y}=0
$$


and the general solution is

$$
v=f(t-y)+g(t+y),
$$

the two functions being arbitrary. Now $h$ is given by (3.4), and so $h=0$ demands that $\gamma^{\prime} / \gamma^{2}=1,1 / \gamma=a-y$ where $a$ is a constant, and so by (3.5)

$$
\begin{aligned}
c^{\prime} / c & =2 \gamma=2 /(a-y), \\
c & =b /(a-y)^{2},
\end{aligned}
$$

where $b$ is a positive constant. Now the $(x, y)$ transformation (2.1) may also be written

$$
d x=c d y=b d y /(a-y)^{2},
$$

and so

$$
x=k+b /(a-y), \quad a-y=b /(x-k),
$$

where $k$ is another constant. Thus by (5.3)

$$
c=b^{-1}(x-k)^{2} \text {. }
$$

But as in (2.2) $c(y)=s(x)$, and so we make $h=0$ by choosing a string for which the local speed of propagation is

$$
s(x)=b^{-1}(x-k)^{2},
$$

where $b$ and $k$ are arbitrary constants with $b$ positive.

For such a string the general solution is as in (5.2): to express the physical displacement $u$ in terms of the physical distance $x$ from the origin, we are to use (5.5) which gives

$$
y=a-b /(x-k)
$$

and so

$$
u=c^{1 / 2} v=b^{-1 / 2}(x-k)\{f(t-a+b /(x-k))+g(t+a-b /(x-k))\}
$$

the functions $f$ and $g$ being arbitrary. It is easy to verify that this satisfies (1.1) with $s(x)$ as in (5.7).

It was said at the outset that the string was infinite, and so it may be objected that (5.9) has a singularity at $x=k$. However, the infinite length of the string was never invoked in the argument, and, even if the length is finite, all the theory holds good until such time as a local disturbance reaches an end. For a string of the special construction as above, a disturbance initiated at or near $x=0$ would require an infinite time to reach $x=k$, because, with local speed as in (5.7), the time taken would be

$$
b \int_{0}^{k}(x-k)^{-2} d x
$$

which is infinite.

6. Conclusion. If the formula (4.8) is regarded as a solution of the problem of the transverse vibrations of a heterogeneous string, then the problem is solved. Rayleigh was interested in that problem, but concentrated on the frequencies of a finite heterogeneous string. Since the final result is so simple, it would be surprising if no one had obtained it; 
but, while I have made no systematic search through the relevant literature, it seems that it has not been hitherto obtained. The present method evolved after many discussions with my colleague, Professor J. T. Lewis, and my debt to him is great, for it was he who recommended (but for different reasons) the transformation $u=v c^{1 / 2}$ instead of $u=v c$ which I had been using. He has developed a different approach, which I hope will be published.*

I wish to thank also several correspondents: Professor A. M. Arthurs, Dr. E. Saibel and Dr. Vincent Hart-not to mention those who have encouraged me by telling me that they did not know of any solution.

Editor's Note: Through an oversight, Professor Synge's paper was not also published in the January 1981 issue, although it was submitted about a month before Professor Lewis'. We regret the error.

* It has since been published in this Quarterly (38, 461-468 (1981)). 